\title{
Reduction of Graphene Oxide by Laser Scribing in Different Atmospheres and Application in Humidity Sensing
}

\author{
Vittorio Scardaci, ${ }^{1}$ Luca Fichera, ${ }^{1}$ Maria E. Fragalà, ${ }^{1}$ Nunzio Tuccitto, ${ }^{1,2}$ Giovanni Marletta, ${ }^{1}$ \\ and Giuseppe Compagnini ${ }^{1}{ }^{1}$ \\ ${ }^{1}$ Dipartimento di Scienze Chimiche, Università di Catana, Viale A.Doria 6 Catania 95125, Italy \\ ${ }^{2}$ CSGI, Università di Catania, Viale A.Doria 6 Catania 95125, Italy \\ Correspondence should be addressed to Giuseppe Compagnini; gcompagnini@unict.it
}

Received 16 June 2020; Accepted 22 September 2020; Published 5 October 2020

Academic Editor: David Cornu

Copyright (c) 2020 Vittorio Scardaci et al. This is an open access article distributed under the Creative Commons Attribution License, which permits unrestricted use, distribution, and reproduction in any medium, provided the original work is properly cited.

\begin{abstract}
Laser scribing, by far proposed as a method for efficiently transforming graphene oxide into reduced graphene, has been employed, using a CW laser beam, to study the modifications of graphene oxide scribed under different controlled atmospheres, including air, $\mathrm{N}_{2}$, Ar, and a 95:5 $(v / v) \mathrm{Ar} / \mathrm{H}_{2}$ mixture. We have found that laser scribing effects, including chemical composition, order degree, and electrical properties, strongly depend upon the atmosphere during the irradiation. The electrical properties of the various materials, obtained in different atmospheres, have been evaluated with respect to the sensing properties towards humidity, the materials produced in pure Argon yielding the highest sensitivity and the fastest response, while related Raman microspectroscopy measurements showed that both $\mathrm{Ar}$ and $\mathrm{Ar}-\mathrm{H}_{2}$ mixture produce the highest order among the various samples. The results stress the importance and the potentialities of controlling the irradiation atmosphere in order to tune the composition and electrical properties of the laser-scribed graphene oxide materials.
\end{abstract}

\section{Introduction}

Since its discovery, graphene has found manifold potential applications due to its remarkable electronic, optical, chemical, and mechanical properties. A wide range of production methods have been developed over time, including both top-down and bottom-up approaches [1]. While bottom-up methods are based on the synthesis of graphene from sources of Carbon, top-down methods aim at having graphene available in large quantities in a liquid medium starting from graphite, its parent material from which it can be exfoliated [1]. Indeed, graphene monolayers can be exfoliated and isolated in organic solvents [2]. Graphene monolayers can also be exfoliated and isolated in water but necessitate the aid of surfactant molecules or polymers to overcome reaggregation due to the strong van der Waals interaction between layers coming from $\pi-\pi$ stacking [3]. One way to dissolve gra- phene in water without using surfactants or molecules is oxidation. In this way, polar functional groups, like carboxylic, epoxy, or hydroxyl, are introduced in the graphene structure making it compatible with water [4]. As the original structure is modified, the process yields a different material, with different properties, which has been named graphene oxide (GO) $[4,5]$. This can be reduced back in order to partially restore the original properties once the material has been processed [6]. However, the structure is never fully restored; hence, such material has been named reduced GO (rGO), to distinguish from the pristine graphene.

Laser irradiation was initially proposed as a mean to pattern GO surfaces [7]. The high thermal energy conveyed by the laser selectively etched the irradiated zones, leaving the unexposed zones intact. Such zones could then be chemically reduced, leaving an $\mathrm{rGO}$ pattern [7]. The same process carried out under $\mathrm{N}_{2}$ atmosphere leads to GO reduction rather 
than etching [7]. Later, Sokolov et al. [8] reduced a GO surface by laser irradiation in both air and $\mathrm{N}_{2}$, noting that the process in $\mathrm{N}_{2}$ produced a more highly ordered $\mathrm{rGO}$ structure.

Such reduction process happens by thermal effect and causes the elimination of $\mathrm{H}_{2} \mathrm{O}, \mathrm{CO}$, and $\mathrm{CO}_{2}$ from the structure $[7,8]$. When GO is deposited on a suitable surface, by a simple drop-casting procedure, the method is particularly attractive as can be used for rapid and precise mask-less patterning, at the micrometric scale, to create conductive rGO traces, surrounded by insulating GO, with no need of photolithography [9-14]. Among several possible uses, such conductive structures have been exploited for resistive humidity sensing $[15,16]$ and mechanical sensing [17], or as electrodes in capacitive humidity sensing $[15,18]$, where the dielectric is the nonreduced GO.

Although Raman spectroscopy has been extensively used to characterize such rGO structures obtained from GO by laser reduction $[13,15,18-20]$, no study has been published to date, to our knowledge, that investigates the uniformity of the process on a mesoscopic scale and the efficiency of the reduction process in different types of atmospheres. In this paper, therefore, we study the reduction of GO films by laser scribing under air as well as inert and slightly reducing atmospheres. We characterize the reduction process and the uniformity of such materials by Raman spectroscopy and X-ray photoelectron spectroscopy (XPS). Furthermore, we also test the response of the fabricated structures against resistive humidity sensing.

\section{Materials and Methods}

GO has been purchased as a $0.4 \mathrm{wt} \%$ water dispersion from Graphenea. The solution has a monolayer content $>95 \%$. An aliquot of such solution is ultrasonicated in a bath for 30 minutes and then drop-casted onto a poly(ethyleneterephtalate) (PET) substrate, which was previously cleaned. The GO solution on PET is left to dry for two days.

Laser scribing has been performed using a Qiilu DK-BL machine at $\sim 6 \mathrm{~mm} / \mathrm{s}$ scan rate with a $405 \mathrm{~nm}$ laser at $1.5 \mathrm{~W}$ power. Four different atmospheres have been used to carry out the scribing procedure: air, Nitrogen, Argon, and a 95:5 Argon $/ \mathrm{H}_{2}$ mixture. Scribing in non-air atmospheres was carried out inside a portable glove box (AtmosBag), flushed with the relevant gas.

The Raman spectroscopy analysis on the obtained structures has been performed with a WITec Alpha 300 RS instrument, using a $600 / \mathrm{mm}$ grating and a $532 \mathrm{~nm}$ laser excitation. Scanning electron microscopy (SEM) was used to characterize sample morphology (Zeiss SUPRA 55-VP). XPS spectra were measured at $45^{\circ}$ take-off angle relative to the surface plane with a PHI 5600 Multi Technique System (base pressure of the main chamber $1 \times 10^{-8} \mathrm{~Pa}$ ). Samples were excited with $\mathrm{Al} \mathrm{K} \alpha \mathrm{X}$-ray radiation using a pass energy of $5.85 \mathrm{eV}$. The instrumental energy resolution was $\leq 0.5 \mathrm{eV}$. Signals due to the $\mathrm{K} \alpha_{1,2}$ and $\mathrm{K} \alpha_{2,3}$ satellite radiations were subtracted from the spectra prior to data processing.

Resistance response to the environmental relative humidity $(\mathrm{RH})$ was verified with an in-house arranged experimental setup, displayed in Figure S1. It consists of a chamber (11) having controlled humidity in which the light-scribed DUT has been placed. The humidity was modulated by blowing in humid nitrogen obtained by varying the relative flow rate of two streams. One stream of nitrogen saturated with water (for bubbling in distilled water) mixed with another flow of dry nitrogen. The typical flow rate was $10 \mathrm{ml} / \mathrm{min}$. Source measure unit (Xtralien X100, Ossila, UK) was used to measure stripes resistance. $\mathrm{RH}$ was monitored by means of a temperature and humidity sensor (sht10, Sensirion). A python ${ }^{\mathrm{TM}}$-based script was used to control the devices and to store the data acquired. All measurements were carried out using a $2 \mathrm{~V}$ voltage-bias applied.

\section{Results and Discussion}

Figure 1(a) represents a scheme of a typical sample layout used for this work. This is made of five stripes ending with two pads for electric contacts, with an overall length around $20 \mathrm{~mm}$ to match the size and shape of the chip carrier. Such structure will be produced by laser scribing and used for resistive sensing measurements. The laser is scanned along the stripe direction, and each stripe is made of two adjacent laser scribing lines, where the laser passes twice. The stripes are around $250 \mu \mathrm{m}$ wide for samples produced in air and around $350 \mu \mathrm{m}$ otherwise, as can be seen by lowmagnification SEM images (Figure S2). We reckon a laser spot size of around $50 \mu \mathrm{m}$. A photographic image of the typical structure is shown in Figure 1(b), where we can observe a uniform dark GO layer formed onto PET, and the five laser-converted darker structures, created by scanning the laser across the GO film.

Figure 2 shows typical SEM images of laser-irradiated samples. It can be observed that the GO film is swollen where modified by the laser (Figure 2(a)). This is caused by the removal of the oxygen-containing functional groups (including hydroxylic, carbonylic, and carboxylic moieties), desorbed as $\mathrm{CO}_{2}$ and $\mathrm{H}_{2} \mathrm{O}[7,8]$. Looking more closely at the modified regions, we can observe a "pastry-like" morphology, with air gaps between the layers, which appear very thin (Figures 2(b) and 2(c)), as also observed previously [14, 15]. Noteworthy, sample morphology does not show significant differences across the different processing atmospheres.

Figure 3 shows Raman spectra from laser-converted GO in four different atmospheres: air, Nitrogen, Argon, and a 95:5 Ar: $\mathrm{H}_{2}$ mixture. The spectrum of pristine $\mathrm{GO}$ is also shown for comparison. The difference between pristine GO and laser-irradiated GO, under any atmosphere, is clear. The GO spectrum shows two very broad features in the $1200-1700 \mathrm{~cm}^{-1}$ region, commonly identified as D- and Gbands, and is flat in the higher wavenumber region. The Dband arises from the breathing mode of aromatic rings in carbon structures and is forbidden by the fundamental $q=0$ Raman selection rule [21]. This is only activated in the presence of structural disorder [21], as is the case for GO. The Gband arises from the stretching of C-C bonds and is typical of all graphite-derived structures [21]. On the other hand, laserirradiated regions, regardless of the atmosphere, show two well-separated G- and D-bands around 1580 and $1350 \mathrm{~cm}^{-1}$, respectively. Moreover, they show an additional peak just 


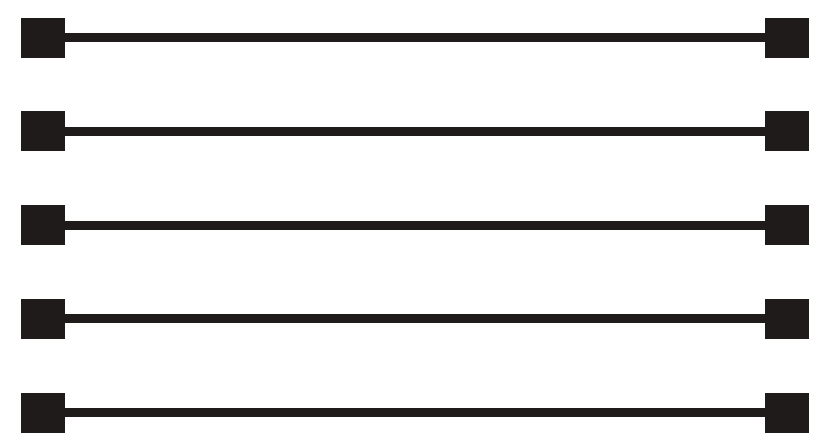

(a)

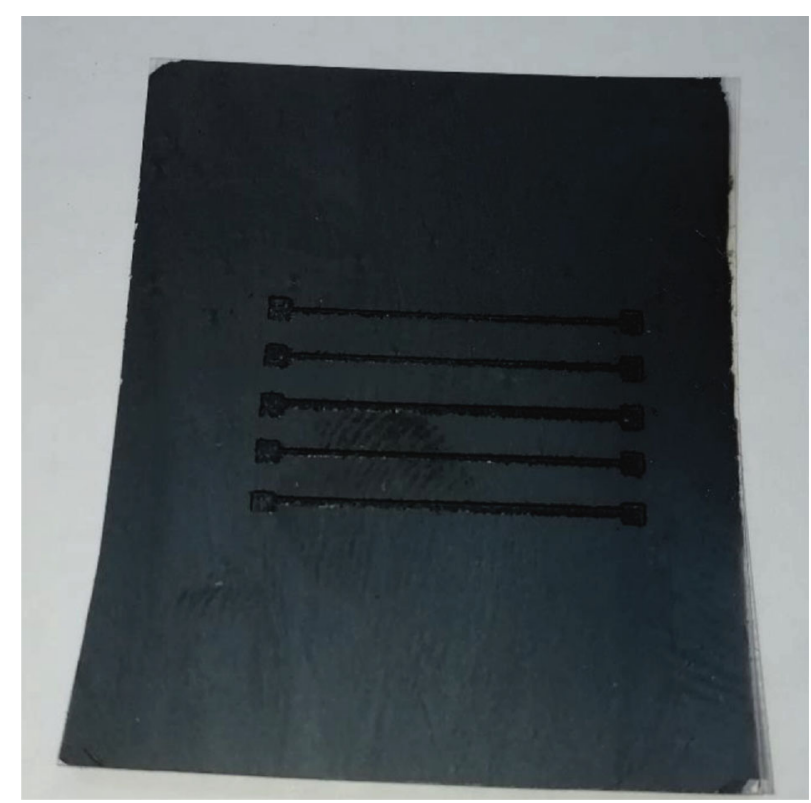

(b)

FIgURE 1: (a) sample layout scheme; (b) photographic image of a typical sample produced by laser scribing of a GO film.

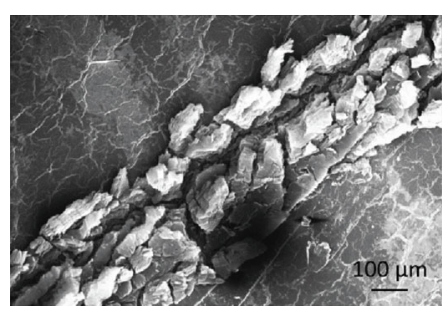

(a)

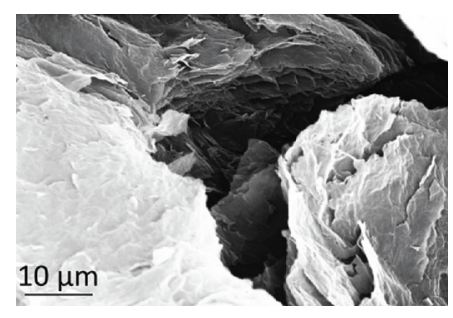

(b)

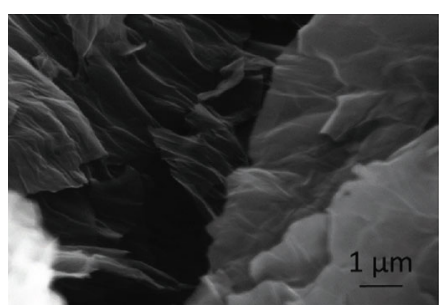

(c)

Figure 2: Typical SEM images of laser-irradiated GO films. (a) Low-magnification image showing the entire line width; (b) mediummagnification image showing the exfoliated structure formed after laser reduction; (c) high-magnification image showing thin RGO layers.

below $2700 \mathrm{~cm}^{-1}$. This is usually indicated as $2 \mathrm{D}$ as is the second order of the D-band and is allowed by selection rules, unlike the first-order D-band [22]. When both the G- and 2D-band show up as single features, as in this case, this is a clear fingerprint of monolayer graphene and thus an unequivocal indication that our laser scribing process is able to produce rGO from GO [22]. This is despite layers appearing stacked, as shown by morphology analysis in Figure 2, suggesting that no electronic interactions occur between the stacked layers, since the Raman spectrum would be dramatically different even if two layers were to interact with each other [22].
A practical way of assessing the structural quality of Carbon structures and compare them is reporting the intensity ratio of the D-band to G-band, commonly referred to as $I_{\mathrm{D}} /$ $I_{\mathrm{G}}$, as a lower $I_{\mathrm{D}} / I_{\mathrm{G}}$ value is associated with a higher degree of order [21]. It has to be noted that in mechanically exfoliated graphene, there is no D-band, and thus, the $I_{\mathrm{D}} / I_{\mathrm{G}}$ value is zero [22]. It is clear from Table 1 that Argon and Argon/Hydrogen atmospheres provide the best-quality rGO, compared to air and Nitrogen.

It must be noted that spectra shown in Figure 3 and $I_{\mathrm{D}} / I_{\mathrm{G}}$ data reported in Table 1 are from the best spectra recorded in each sample. While such structures are macroscopic in size, 


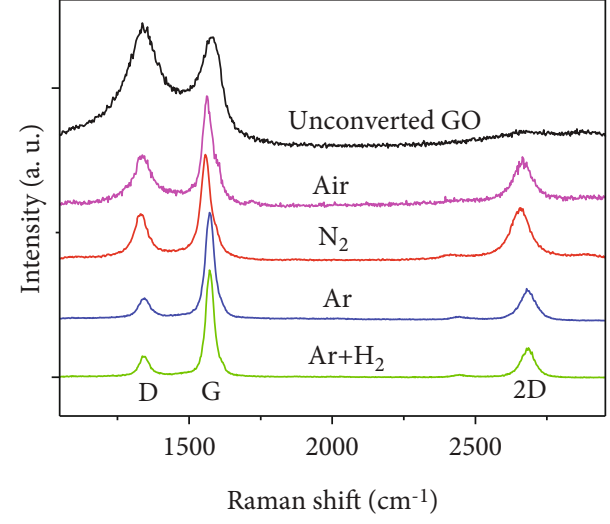

Figure 3: Raman spectra from rGO stripes produced under different atmospheres and unconverted GO.

TABLE 1: $I_{\mathrm{D}} / I_{\mathrm{G}}$ values from the best spectra in different atmospheres.

\begin{tabular}{lc}
\hline Atmosphere & $I_{\mathrm{D}} / I_{\mathrm{G}}$ \\
\hline Air & 0.44 \\
Nitrogen & 0.43 \\
Argon & 0.19 \\
Argon/Hydrogen & 0.19 \\
\hline
\end{tabular}

the Raman spectroscopy can only probe a limited area, as big as the laser spot size, typically no more than a few microns in diameter. Thus, we have randomly taken multiple spectra from each sample, in order to compare the $I_{\mathrm{D}} / I_{\mathrm{G}}$ distributions and draw a clearer picture of material uniformity. Figure 4 shows, for each atmosphere, $I_{\mathrm{D}} / I_{\mathrm{G}}$ values in ascending sequences for each atmosphere. Accordingly, a uniform sample would show a nearly horizontal data trend, regardless of the $I_{\mathrm{D}} / I_{\mathrm{G}}$ value, while a heterogeneous sample would show the highest slope. Hence, it appears obvious that none of the samples is properly homogeneous, but the closest to uniformity seems to be the ones produced in Argon, with most $I_{\mathrm{D}} /$ $I_{\mathrm{G}}$ values in the region of 1 . It is also clear that the $\mathrm{Ar} / \mathrm{H}_{2}$ atmosphere shows a larger amount of spectra with a low $I_{\mathrm{D}} / I_{\mathrm{G}}$ compared to Ar. Hence, while the best spectra are comparable, the overall data distribution suggests that $\mathrm{Ar} / \mathrm{H}_{2}$ is a better atmosphere to produce rGO from GO.

Comparing the samples obtained by laser irradiation in $\mathrm{N}_{2}$ with those irradiated in air, the curve for samples in air shows a large number of spectra with very high $I_{\mathrm{D}} / \mathrm{I}_{\mathrm{G}}$, i.e., vast zones where GO was not converted, thus suggesting that air is a worse environment than $\mathrm{N}_{2}$. These findings then suggest that in terms of rGO quality, the irradiation atmospheres rank as $\mathrm{Ar}-\mathrm{H}_{2}>\mathrm{Ar}>\mathrm{N}_{2}>$ air. Air contains $\sim 20 \% \mathrm{O}_{2}$ and as such is a mild oxidizing environment, so it is not surprising that our reduction process results less effective in such environment than inert atmospheres. Likewise, a slightly reducing environment such as one containing $5 \% \mathrm{H}_{2}$ results more effective than an inert one in reducing GO. As for the differences observed between two inert atmospheres like Nitrogen and Argon, the origin is not clear. This effect is also

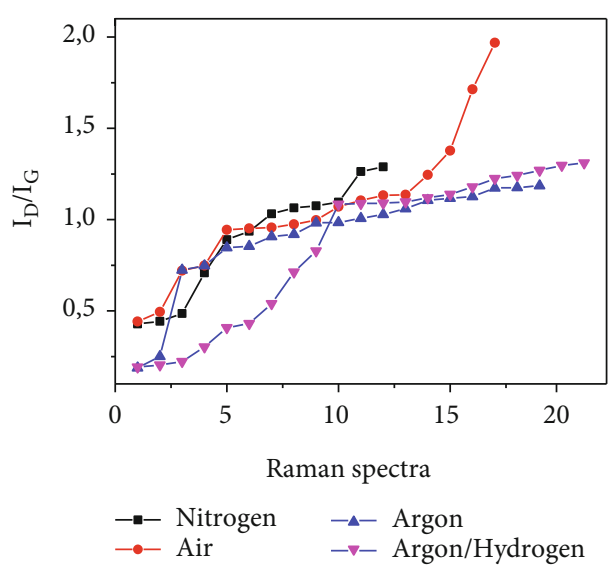

FIgURE 4: $I_{\mathrm{D}} / I_{\mathrm{G}}$ values in ascending sequence for different atmospheres.

confirmed by XPS analysis (Figure S3). Here, while in the GO area, we observe a double feature in the $\mathrm{C}$ area, attributed to $\mathrm{C}=\mathrm{C}$ and $\mathrm{C}-\mathrm{O}-\mathrm{C}$ bonds, in the $\mathrm{RGO}$ area, we observe only the $\mathrm{C}=\mathrm{C}$ peak, with the loss of the $\mathrm{C}-\mathrm{O}-\mathrm{C}$ one.

The laser-irradiated samples show a marked change of the electrical properties, which, in turn, are affected by exposure to humidity. Figure 5 shows the effect of humidity on the relative resistance, intended as $\Delta R / R_{0}$, measured at various $\mathrm{RH}$ values on rGO produced under different atmospheres. All samples show a linear response up to $10-20 \% \mathrm{RH}$. Above this value, any $\mathrm{RH}$ increase does not affect the relative resistance of the samples. Noteworthy, the environmental conditions in which the laser irradiation of GO was performed strongly influence the response of the various samples in terms of slope and threshold value of the plateau. This seems to indicate a variation of the physicochemical structure and composition of the GO induced by the irradiation atmosphere. We fitted the experimental data shown in Figure 5 with the equation:

$$
\frac{\Delta R}{R_{0}}=j \cdot\left(1-e^{-k \cdot \mathrm{RH}}\right)
$$

where $j$ is the plateau value while the fitting parameter $k$ is an indication of the speed at which the sensor reaches the plateau. Based on the values obtained, we found that the samples prepared in Argon is the most responsive to moisture, while the one prepared in air is the least responsive. Figure 6 shows a hysteresis plot reporting the variation of resistance against measured $\mathrm{RH}$. Such plot indicates how the response of our material reflects actual $\mathrm{RH}$ (measured by a commercial sensor). The trace relative to Argon shows nearly no hysteresis, suggesting a response very close to ideal. Figure 6 inset shows the two responses from the material prepared in Argon and the commercial sensor plotted against time. Indeed, such responses are identical when $\mathrm{RH}$ increases, while very little delay appears in our material when $\mathrm{RH}$ recovers towards zero. On the other hand, the material prepared in Nitrogen shows the maximum hysteresis, carrying the slowest response with both 


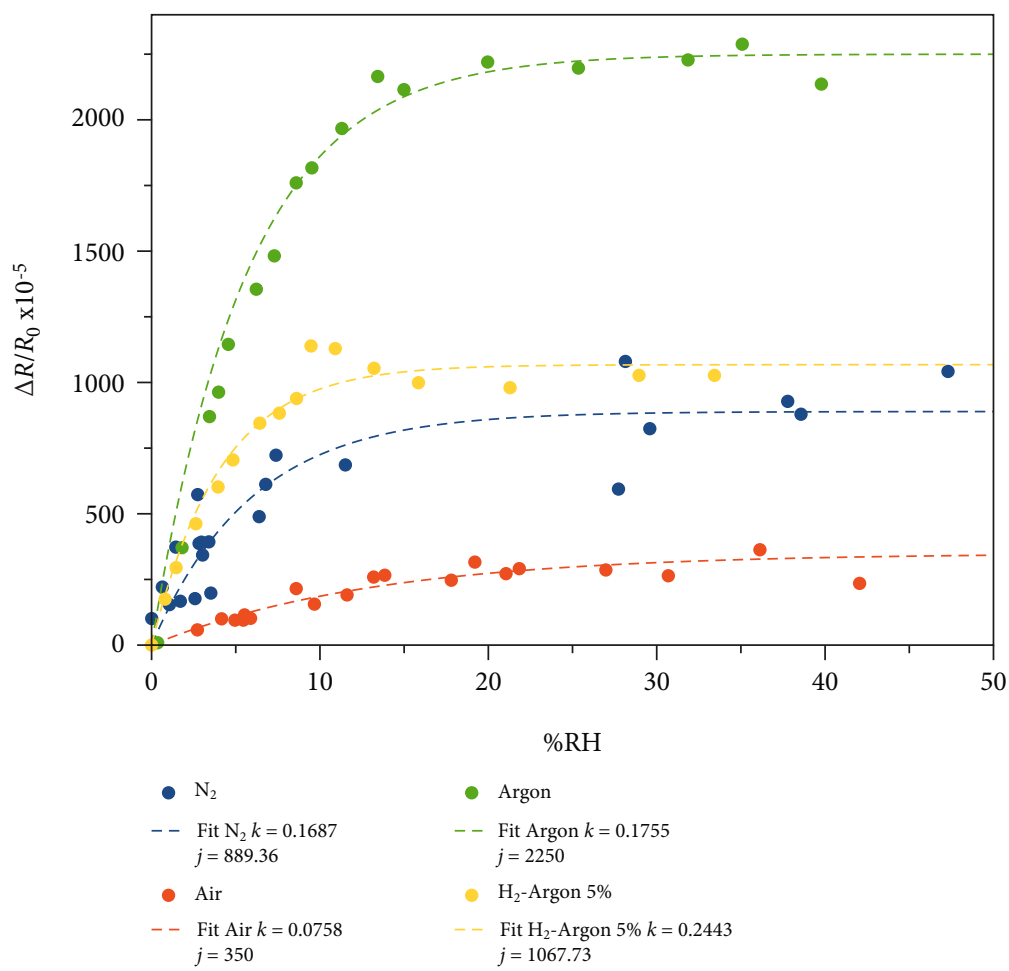

FIGURE 5: RGO resistive response to repeated RH exposure cycles under different atmospheres.

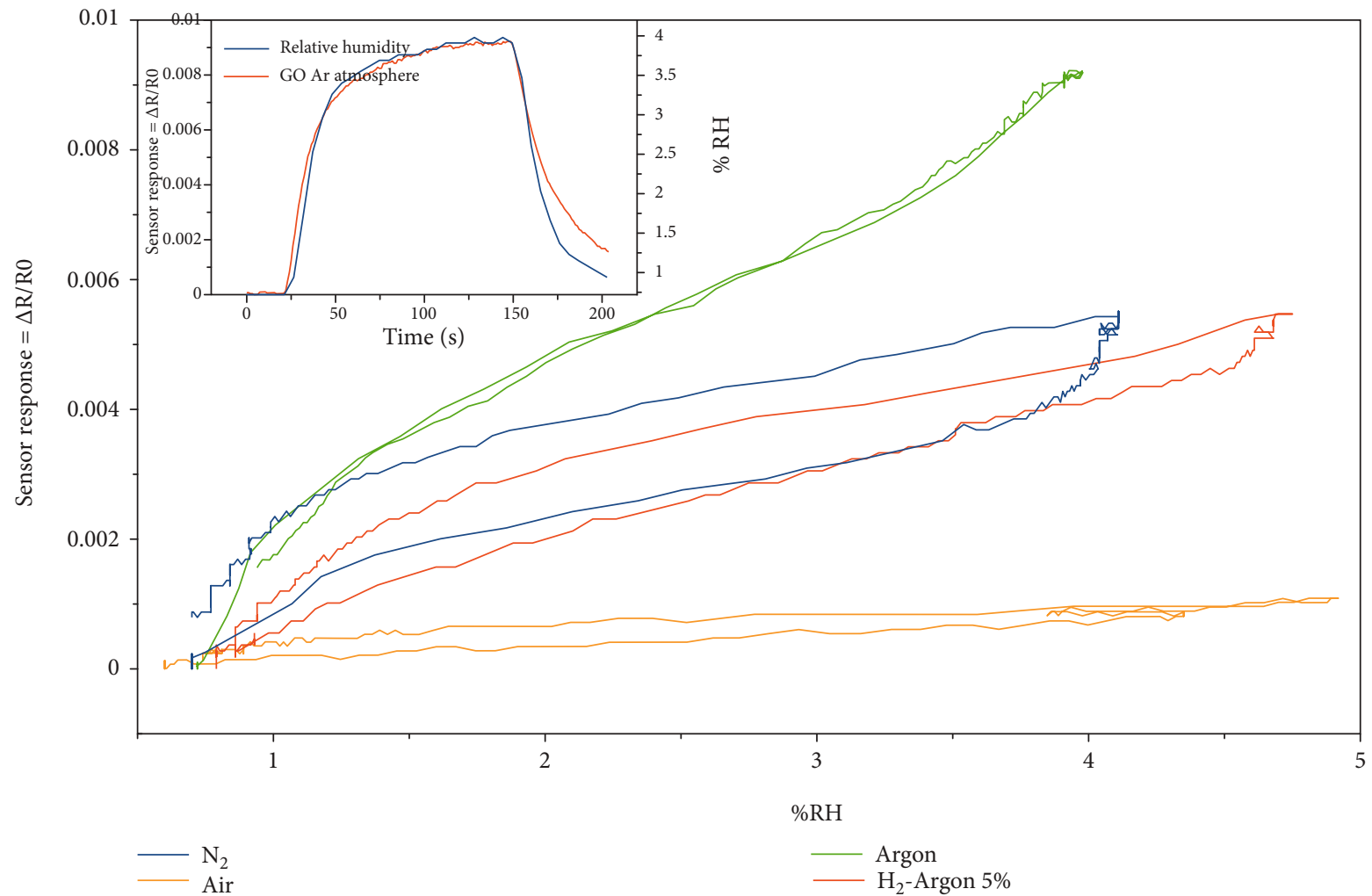

FiguRE 6: RGO response to a single RH exposure cycle under different atmospheres. Inset: parallel representation of RH measured by a commercial sensor and the response from rGO produced in Argon. 
increasing and decreasing RH (see Figures S4-S6). Results confirm that the samples fabricated in air show the worst response to $\mathrm{RH}$ variations, while the one produced in Argon responds more readily to changes in $\mathrm{RH}$ with no hysteresis.

While the current measured for the RGO stripes was in the region of tens of $\mu \mathrm{A}$ (for a $2 \mathrm{~V}$ applied voltage), the current for unconverted GO was in the range of 5 orders of magnitude lower being actually insulator. On the other hand, the laser-converted material is conductive and porous, allowing by swelling $\mathrm{H}_{2} \mathrm{O}$ molecules to interact with a larger surface area. Thus, resistance increases as $\mathrm{RH}$ increases. This is usually explained by different mechanisms. At low $\mathrm{RH}, \mathrm{H}_{2} \mathrm{O}$ molecules are adsorbed onto the RGO through the doubleelectron pair on oxygen, forming hydrogen bonds with oxygen-containing functional groups onto the surface [2325]. This suggests a p-type semiconductor behavior, in agreement with other reports. At higher $\mathrm{RH}$ levels, further $\mathrm{H}_{2} \mathrm{O}$ adsorption causes the structure to swell $[23,25]$, making carrier hopping between adjacent flakes harder, i.e., increasing junction resistance, thus increasing the overall resistance. However, as our structure is already swollen due to the laser scribing process, the latter mechanism may have a limited importance, and we observe resistance increase only to some extent.

The sensitivity differences between RGO obtained in different atmospheres arise from a combination of effects. Since samples produced in $\mathrm{Ar}-\mathrm{H}_{2}$ show a higher conversion to RGO, they have less oxygen-containing residual functional groups than samples produced in Argon. This allows the latter material to have a better interaction with $\mathrm{H}_{2} \mathrm{O}$ molecules in the nearby environment, leading to a higher sensitivity. Samples produced in $\mathrm{N}_{2}$ are significantly different from those produced in Ar, despite $\mathrm{N}_{2}$ being as inert. This is an open question at the moment. Samples produced in air show areas of unconverted material, as seen by the Raman spectroscopy, forming a surface that is highly inhomogeneous from an electrical conductivity standpoint, causing a low overall sensitivity to $\mathrm{H}_{2} \mathrm{O}$ molecule adsorption.

\section{Conclusions}

We used a laser scribing process under different atmospheres to convert GO deposited on a surface to rGO. The Raman spectroscopy confirmed that the process yields monolayer graphene in any environment and shows that the bestquality rGO is obtained in a mixture of $\mathrm{Ar}(95 \%)$ and $\mathrm{H}_{2}$ (5\%) as scribing environment, while the most uniform is obtained in Ar. XPS measurements confirm the trend. Humidity sensing tests show that the material prepared in Ar has the highest sensitivity to $\mathrm{RH}$ variations at low $\mathrm{RH}$ levels, and the lowest response delay compared to a commercial sensor.

\section{Data Availability}

Data are available from the authors upon request.

\section{Conflicts of Interest}

The authors declare that there is no conflict of interest regarding the publication of this paper.

\section{Acknowledgments}

Funding was received from Ministero della Università e della Ricerca (MIUR) (Project ARS01_00519-BEST4U), Piano della Ricerca di Ateneo 2020-22, and Biotechnology Research and Innovation Tower (BRIT).

\section{Supplementary Materials}

Figure S1: this figure represents the setup used to carry out humidity measurements in parallel with resistance measurements. Figure S2: this figure shows low-magnification SEM images of RGO lines made by laser scribing. Figure S3: this figure shows XPS spectra of GO and RGO in Argon atmosphere. Figures S4, S5, and S6: these figures show a parallel representation of RH measured by a commercial sensor and the response from rGO produced in air, nitrogen and Argon- $\mathrm{H}_{2}$, respectively. (Supplementary Materials)

\section{References}

[1] J. M. Tour, "Top-down versus bottom-up fabrication of graphene-based electronics," Chemistry of Materials, vol. 26, no. 1, pp. 163-171, 2014.

[2] Y. Hernandez, V. Nicolosi, M. Lotya et al., "High-yield production of graphene by liquid-phase exfoliation of graphite," Nature Nanotechnology, vol. 3, no. 9, pp. 563-568, 2008.

[3] S. De, P. J. King, M. Lotya et al., "Flexible, transparent, conducting films of randomly stacked graphene from surfactantstabilized, oxide-free graphene dispersions," Small, vol. 6, no. 3, pp. 458-464, 2010.

[4] D. R. Dreyer, S. Park, C. W. Bielawski, and R. S. Ruoff, "The chemistry of graphene oxide," Chemical Society Reviews, vol. 39, no. 1, pp. 228-240, 2010.

[5] Y. Zhu, S. Murali, W. Cai et al., "Graphene and graphene oxide: Synthesis, Properties, and Applications, properties, and applications," Advanced Materials, vol. 22, no. 35, pp. 3906-3924, 2010.

[6] S. Stankovich, D. A. Dikin, R. D. Piner et al., "Synthesis of graphene-based nanosheets via chemical reduction of exfoliated graphite oxide," Carbon, vol. 45, no. 7, pp. 1558-1565, 2007.

[7] Y. Zhou, Q. Bao, B. Varghese et al., "Microstructuring of graphene oxide nanosheets using direct laser writing," Advanced Materials, vol. 22, no. 1, pp. 67-71, 2010.

[8] D. A. Sokolov, K. R. Shepperd, and T. M. Orlando, "Formation of graphene features from direct laser-induced reduction of graphite oxide," The Journal of Physical Chemistry Letters, vol. 1, no. 18, pp. 2633-2636, 2010.

[9] Y. Zhang, L. Guo, S. Wei et al., "Direct imprinting of microcircuits on graphene oxides film by femtosecond laser reduction," Nano Today, vol. 5, no. 1, pp. 15-20, 2010.

[10] M. F. El-Kady and R. B. Kaner, "Scalable fabrication of highpower graphene micro-supercapacitors for flexible and onchip energy storage," Nature Communications, vol. 4, no. 1, 2013. 
[11] W. Gao, N. Singh, L. Song et al., "Direct laser writing of microsupercapacitors on hydrated graphite oxide films," Nature Nanotechnology, vol. 6, no. 8, pp. 496-500, 2011.

[12] D. A. Sokolov, C. M. Rouleau, D. B. Geohegan, and T. M. Orlando, "Excimer laser reduction and patterning of graphite oxide," Carbon, vol. 53, pp. 81-89, 2013.

[13] R. Trusovas, K. Ratautas, G. Račiukaitis et al., "Reduction of graphite oxide to graphene with laser irradiation," Carbon, vol. 52, pp. 574-582, 2013.

[14] M. F. El-Kady, V. Strong, S. Dubin, and R. B. Kaner, "Laser scribing of high-performance and flexible graphene-based electrochemical capacitors," Science, vol. 335, no. 6074, pp. 1326-1330, 2012.

[15] J. Cai, C. Lv, E. Aoyagi, S. Ogawa, and A. Watanabe, "Laser direct writing of a high-performance all-graphene humidity sensor working in a novel sensing mode for portable electronics," ACS Applied Materials \& Interfaces, vol. 10, no. 28, pp. 23987-23996, 2018.

[16] A. A. Semenova, P. V. Vinokurov, and S. A. Smagulova, "Investigation of the properties of flexible humidity sensor based on reduced graphene oxide by laser irradiation," in AIP Conference Proceedings, vol. 2041, article 020017, Yakutsk, Russia, 2018.

[17] Y.-C. Qiao, Y.-H. Wei, Y. Pang et al., "Graphene devices based on laser scribing technology," Japanese Journal of Applied Physics, vol. 57, no. 4S, article 04FA01, 2018.

[18] F. J. Romero, A. Rivadeneyra, A. Salinas-Castillo et al., "Design, fabrication and characterization of capacitive humidity sensors based on emerging flexible technologies," Sensors and Actuators B: Chemical, vol. 287, pp. 459-467, 2019.

[19] A. Longo, R. Verucchi, L. Aversa et al., "Graphene oxide prepared by graphene nanoplatelets and reduced by laser treatment," Nanotechnology, vol. 28, no. 22, 2017.

[20] C.-R. Yang, S.-F. Tseng, and Y.-T. Chen, "Laser-induced reduction of graphene oxide powders by high pulsed ultraviolet laser irradiations," Applied Surface Science, vol. 444, pp. $578-583,2018$

[21] A. C. Ferrari and J. Robertson, "Interpretation of Raman spectra of disordered and amorphous carbon," Physical Review B, vol. 61, no. 20, pp. 14095-14107, 2000.

[22] A. C. Ferrari, J. C. Meyer, V. Scardaci et al., "Raman spectrum of graphene and graphene layers," Physical Review Letters, vol. 97, no. 18, article 187401, 2006.

[23] Y. Yao, X. Chen, J. Zhu, B. Zeng, Z. Wu, and X. Li, “The effect of ambient humidity on the electrical properties of graphene oxide films," Nanoscale Research Letters, vol. 7, no. 1, 2012.

[24] H. Bi, K. Yin, X. Xie et al., "Ultrahigh humidity sensitivity of graphene oxide," Scientific Reports, vol. 3, no. 1, 2013.

[25] A. De Luca, S. Santra, R. Ghosh et al., "Temperature-modulated graphene oxide resistive humidity sensor for indoor air quality monitoring," Nanoscale, vol. 8, no. 8, pp. 4565-4572, 2016. 